\title{
Diffractive and Holographic Optics as Optical Combiners in Head Mounted Displays
}

\section{Bernard C. Kress}

Google Inc.

1600 Amphitheatre Prkw

Mountain View, CA 94043

bkress@google.com

Meimei Shin

Nanoptron Inc.

548 Keelson Circle

Redwood City, CA 94065

info@nanoptron.com
Permission to make digital or hard copies of all or part of this work for personal or classroom use is granted without fee provided that copies are not made or distributed for profit or commercial advantage and that copies bear this notice and the ful citation on the first page. Copyrights for components of this work owned by others than ACM must be honored. Abstracting with credit is permitted. To copy otherwise, or republish, to post on servers or to redistribute to lists, requires prior specific permission and/or a fee. Request permissions from permissions@acm.org

\begin{abstract}
We review in this paper the various architectures that have been developed in industry to implement seethrough head-mounted display (HMD) optics, especially for the consumer electronics market. We will focus our investigations on holographic and diffractive optics.
\end{abstract}

\section{Author Keywords}

Head Mounted Displays (HMD), Head Up Displays

(HUD), see through display, diffractive and holographic optics, optical combiners, augmented reality.

\section{ACM Classification Keywords}

H.5.m. Information interfaces and presentation (HCI).

\section{Introduction}

Head-Up Displays (HUD), Helmet- or Head-Mounted Displays (HMDs) as well as gun sights have been extensively investigated during the past decades for military applications by various defense contractors. While the first HMD optical combiner optics have been based on conventional reflective/refractive optics, the first and most efficient HUD combiner technologies have been based rather on holographic optics. We investigate in this paper the various potential HMD optical combiner architectures based on such holographic and/or diffractive optics, and discuss their respective advantages and problems. 


\section{Conventional HMD optical combiners}

There are mainly two different optical architectures when it comes to designing see through (or immersive) optics for HMDs: the pupil forming and the non-pupil forming architectures (see Fig.1), [1], [2], [3].
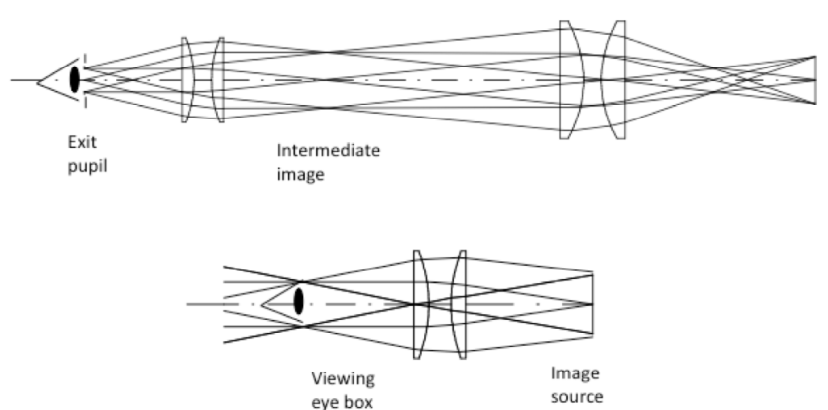

Figure 1. Pupil forming and non-pupil forming HMD optical architectures.

Pupil forming architectures are used when the optical train needs to conform to the users' head shape in a wrap-around format. It produces an intermediate image which allows the integration of interesting optical functionalities such as exit pupil expanders (EPE) [5]. It also allows flexibility in the choice of the combiner optics, since custom LCD/LCOS display sizes can be formed in the intermediate image plane. A non pupi forming architecture produces no intermediate image, the micro-display field is directly collimated to produce an image at infinity. While it is a more compact architecture (often used in occlusion HMDs) it is also less flexible. The eye box of non-pupil forming HMDs is usually larger than the one of pupil forming HMDs, therefore requiring the use of an additional EPE (Exit Pupil Expander). Both versions have been widely implemented in see through HMDs [4]. Fig. 2 shows some of the conventional implementations ranging from (left to right and top to bottom): 45 degree 50/50 or PBS (Polarization Beam Combiner), tilted on-axis flat diffractive lens, tilted linear grating (no power), tilted off-axis diffractive lens, "bug-eyed" reflective lens, Fresnel type lens (segmented), TIR multi surface free form collimator and multiple mirrors light-guide combiners.
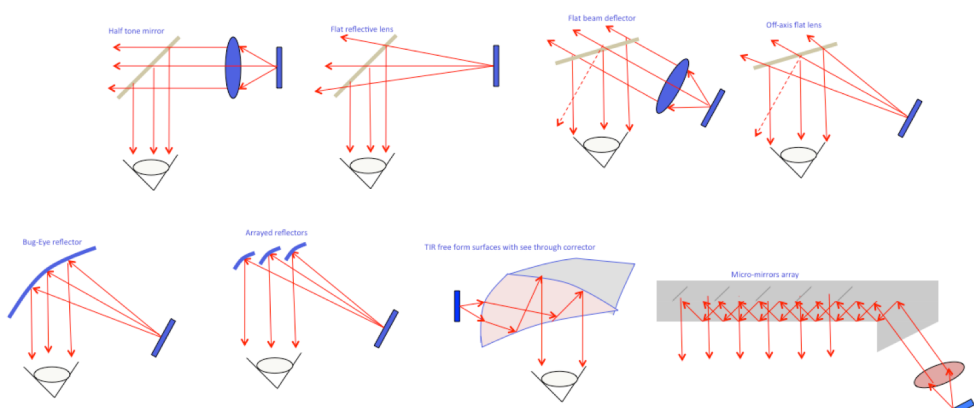

Figure 2. Conventional implementations of combiners.

Although such conventional refractive/reflective optics can produce relatively good see-through and imaging qualities, the main problem remains the limited size of the resulting eye box and the thickness of the combiner optic. There is usually a linear relation between these two critical criteria. In order to attempt to reduce the thickness of the combiner and at the same time increase the size of the eye box, (especially in the horizontal directions), non conventional optics such as holographic and diffractive optics have been proposed. 


\section{Holographic combiners: the angular and spectral Bragg selectivity.}

The use of free space volume holographic HUD

combiners has been investigated since the early 70 s (in military avionics). The concept of using a waveguide grating or waveguide hologram to extract progressively a collimated image guided by Total Internal Reflection (TIR) in a waveguide or light pipe has been studied and patented since the early $80 \mathrm{~s}$. The spectral and angular Bragg selectivity is the key functionality sought after, either in free space or waveguide modes, and in either reflection or transmission operations (Fig. 3) [4].

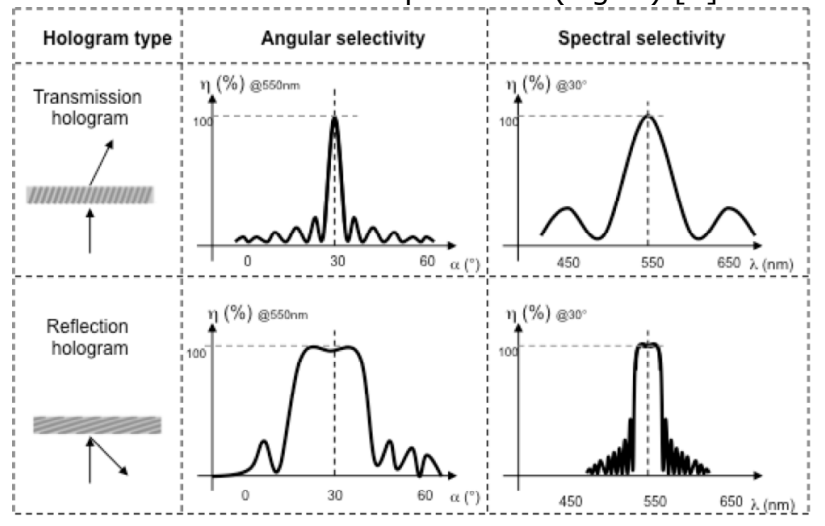

Figure 3. Angular and spectral Bragg selectivity in both transmission and reflection modes.

Fig. 3 shows that the spectral bandwidth is thinner and the angular bandwidth is wider in reflection mode as compared to transmission mode operation. Higher angular bandwidth allows for larger FOV design, and narrower spectral bandwidth allows for operation with broader spectral sources, such as LEDs (the hologram acting as a spectral filter). For example, Sony (Fig. 4.a) and Konica-Minolta (Fig. 4.b) use both reflection volume holograms.
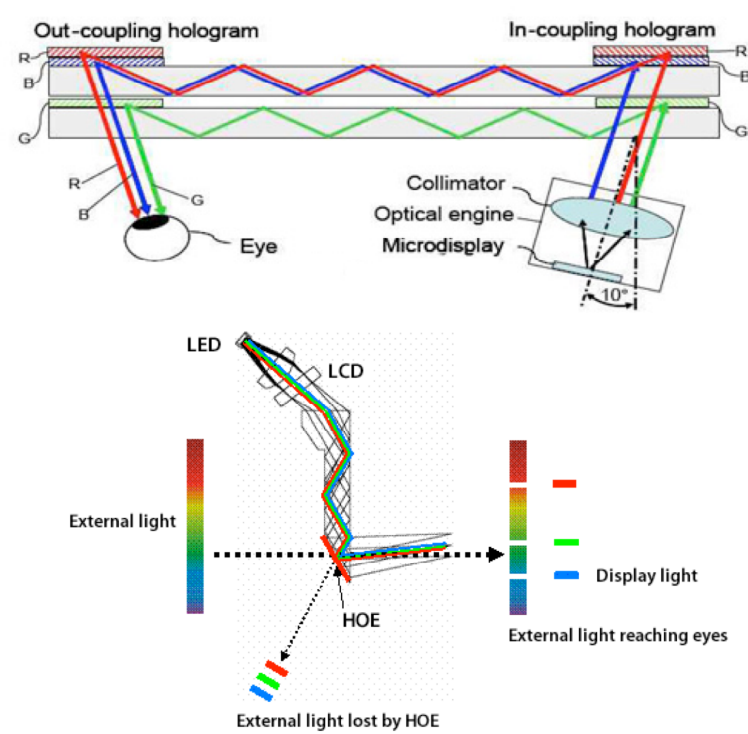

Figure 4. Top: Sony LTD waveguide combiner. Bottom: Konika Minolta LTD reflective holographic combiner.

Reflective volume holographic combiners can produce effective see-through optics, due to their wide angular bandwidth, yielding unaltered see-through quality. Three superimposed holograms, each exposed for a specific wavelength (as in Sony's), or via multiple exposures in the same holographic media (as in Konica/Minolta's) can produce full color RGB holographic combiners to be used with LEDs. In Konica/Minolta's design, the single hologram is used both as a combiner and the collimation lens. Volume holographic combiner architectures seem thus to be exciting technologies for consumer electronics when compared to traditional optical elements. However, their analog nature (volume holographic media such as photopolymers, DCG, silver halides, H-PDLC,...) yield various problems when exposed to environmental 
perturbations such as temperature, humidity or pressure. It became therefore interesting to investigate if such Bragg selectivity could also be achieved in "thin" holograms, (or surface relief diffractives), produced in plastic materials [7].

\section{Diffractive optics as optical combiners}

Nokia attempted to use surface relief diffractive optics to implement similar volume holographic effects [6]. Here the element is not a volume hologram but rather a surface relief diffractive composed of slanted subwavelength relief gratings. Fig 5 (left), shows a transmission in-coupling grating and 2 out-coupling reflective gratings. The waveguide grating on the right side of Fig.5. is at the same time an optical combiner and an 2D EPE. The main problems of such architecture are the complexity of the master fabrication and mass replication as well as the small angular bandwidth (related to the resulting FOV). In order to mimic the holographic Bragg effect, sub-wavelength tilted structures with a high aspect ratio are needed, difficult to mass replicate for low cost volume production [8].

\section{References}

[1] James Melzer "Head Mounted Displays", Mac Graw Hill (1997) (ISBN 978-1456563493).

[2] Mordekhai Velger, "Helmet mounted displays and sights", Artech House- (ISBN 0-89006-822-4).

[3] Clarence E. Rash "Head Mounted Display: design issues for rotary-wing aircraft", US Army Aeromedical Research Laboratory -1999-735-1.

[4] Ozan Cakmakci and Jannick Rolland, "Head-Worn Displays: A Review", Journal of Display Technology, Vol. 2, No. 3, September 2006
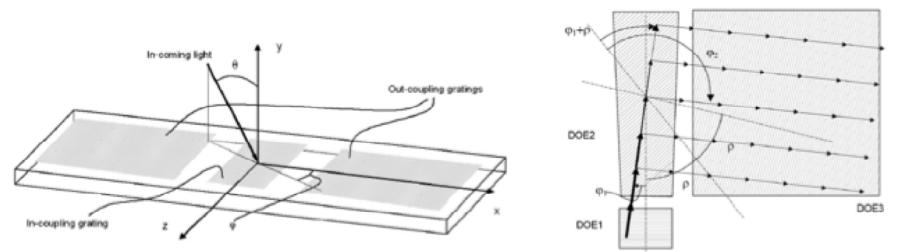

Figure 5. Surface relief slanted sub-wavelength gratings as optical combiners and exit pupil expanders.

\section{Conclusion}

We have reviewed various optics for see through HMDs, from traditional optics to micro-optics, diffractives and holographics. The wide range of optics used by industry today show that there is no standard of optical combiner architecture prevailing, addressing effective functionality for consumer electronics market (i.e. large eye box and FOV, high imaging quality, wrap-around, low environment sensitivity, in a thin form factor).

[5] Hakan Urey "Diffractive exit pupil expander for display applications", Applied Optics Vol. 40, No 21. pp.5840-5851 (2001).

[6] Tapani Levola "Diffractive optics for virtual displays", SID (Society for Image Display), 14/5 (2006)

[7] B. Kress and P. Meyrueis "Applied Digital Optics, from Micro-Optics to Nano-Photonics", John Wiley Publisher, January 2009 (ISBN 9780470022634).

[8] Bernard Kress and Victorien Raulot "Digital combiner achieves low cost and high reliability", SPIE newsroom http://spie.org/x35062.xmlv 